\title{
Nomograms for predicting the overall survival of patients with cerebellar glioma: An analysis of the SEER (Surveillance Epidemiology and End Results) database
}

Jie Li ( $\sim 1548337395 @ q q . c o m$ )

Dalang Hospital

Wobin Huang

Dalang Hospital

Jiajing Chen

Dalang Hospital

Zhuhui Li

Dalang Hospital

Bocong Liu

Dalang Hospital

Peng Wang

Dalang Hospital

Jun Zhang

Dalang Hospital

Research Article

Keywords: Cerebellum glioma, Nomogram, Overall survival, SEER database

Posted Date: July 28th, 2021

DOl: https://doi.org/10.21203/rs.3.rs-741665/v1

License: (c) (i) This work is licensed under a Creative Commons Attribution 4.0 International License.

Read Full License 


\title{
Nomograms for predicting the
}

\section{overall survival of patients with}

\section{cerebellar glioma: An analysis of the} SEER (Surveillance Epidemiology

\section{and End Results) database}

\author{
Jie Li, Wobin Huang, Jiajing Chen, Zhuhui Li, Bocong Liu, Peng Wang, \\ Jun Zhang \\ Affiliations: Neurosurgery, Dalang Hospital, Dongguan, Guangdong, China, 523770. \\ Corresponding Author's name: Jie Li \\ Corresponding Author's Email: 1548337395@qq.com
}

\begin{abstract}
Purpose: At present, our understanding of cerebellar glioma is still not enough. This study collected information on patients in the seer database to determine the predictive factors patients with cerebellar glioma.

Patients and Methods: The data of patients with cerebellar glioma diagnosed from 1975 to 2018 were retrieved from the Surveillance Epidemiology and End Results Database. We randomly divide the data into a training group and a validation group, establish a nomogram based on the training group, and use the validation group data to verify the clinical value of the model.

Results: A total of 508 patients were included in this study. Multivariate analysis was performed based on the data before randomization, and the results showed that the patient's age, the tumor's WHO grade, histological type, and extension are significantly correlated with the survival rate. The $\mathrm{c}$ index of OS nomograms of the training cohort was $0.909(95 \% \mathrm{Cl},(0.880-0.938)$ ) and $0.932(95 \% \mathrm{Cl},(0.889-0.975))$ in validation group. The calibration curve of OS for 3 and 5 years show that there was a good consistency between the actual survival probability and the predicted survival probability.

Conclusion: For patients with cerebellar glioma, the age of the patient at the time of diagnosis, WHO grade of the glioma, histological type, and extension are the four most prominent factors that affect the overall survival outcomes. Furthermore, our model may be a useful tool for predicting OS in these patients.
\end{abstract}

Key words: Cerebellum glioma, Nomogram, Overall survival, SEER database 


\section{Introduction}

Gliomas are common intracranial tumors, but gliomas that occur in the cerebellum are very rare, especially in adults ${ }^{1}$. Because of its rarity, there had few previous studies on this area, so it is necessary to conduct research in this field. AS we known, regardless of the patient's age, histology, or pathology, cerebellar gliomas are serious neurological tumors, usually manifested as life-threatening complications such as compression of the cerebellum and adjacent brainstem or obstruction of the cerebrospinal fluid circulation pathway, resulting in increased intracranial pressure, which leads to serious consequences and even threatens the life of the patient. Some researcher have reported that patients with cerebellar glioblastoma have a poor prognosis, with a survival period of three to seven months ${ }^{2,3}$, while others had observed that cerebellar glioblastoma and brain glioblastoma are similar in survival time ${ }^{4,5}$. In this article, we collected information on patients with cerebellar glioma from the seer database, explored the factors that affect the overall survival time of cerebellar glioma, and constructed a clinical prognostic model of cerebellar glioma to provide risk assessment for clinical patients.

\section{Methods}

\section{Study Population}

The data of patients with cerebellar glioma diagnosed from 1975 to 2018 were retrieved from the Surveillance Epidemiology and End Results Database. The Surveillance Epidemiology and End Results Database collects information about patients' baseline characteristics, and the information of tumor such as histological characteristics, WHO grade, surgery, and patients' vital status in 9 registries within the United States. By using SEER*Stat software (Version 8.3.9), we retrieved 65369 cases in total. The inclusion criteria included 1) first primary cerebellar glioma; 2) The ICD-O-3 site codes were limited to Cerebrum (C71.6); 3) The detailed information on sex, race, and tumor size, WHO grade, surgery information, histological characteristics, pathological diagnosis, and patients' vital status. The exclusion criteria include 1) Patients without histological confirmed glioma; 2) Patients with unknown information such as tumor size, extension, reason for death and surgery status Figure 1. 


\section{Figure1}

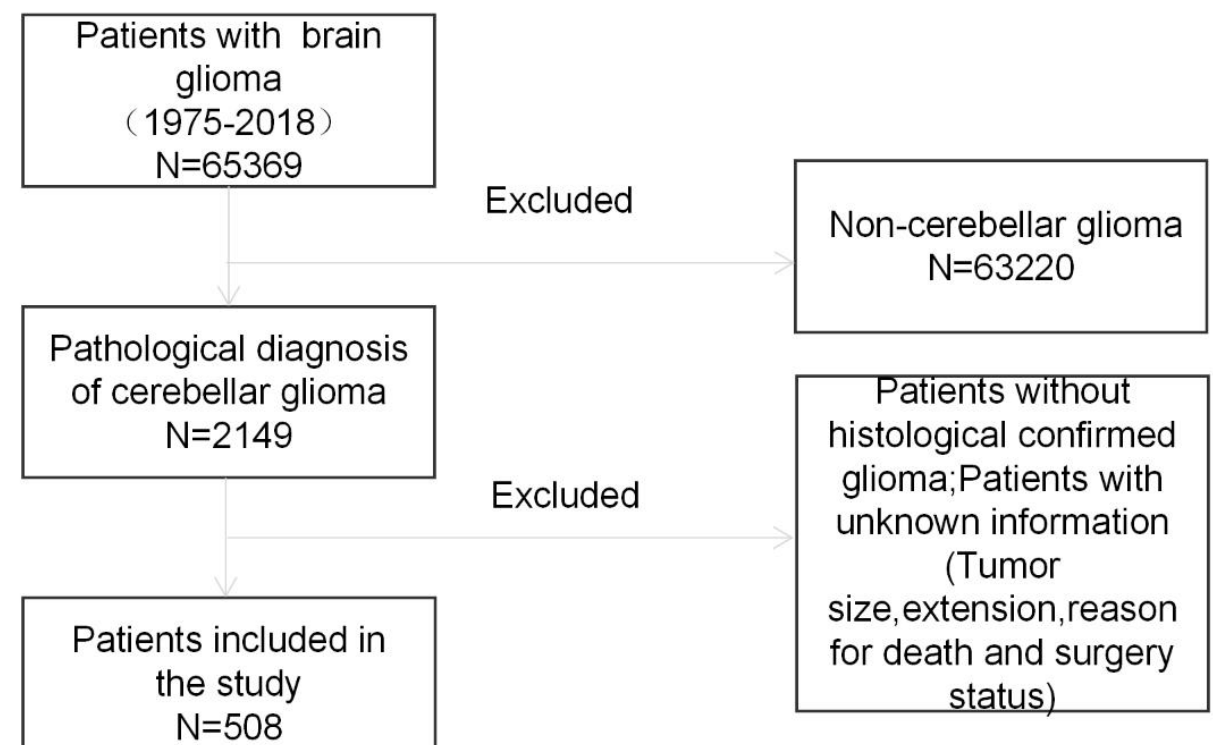

Figure 1. Flowchart of cerebellum glioma patient selection.

\section{Covariates Included}

The data included for analysis were: age ( $\leq 20$ years old were defined as pediatric group, and $>20$ years old were defined as adult group), race (white, black and other) and sex. The cerebellar glioma characteristics data obtained for analysis were: Tumor size (size $\leq 3.0 \mathrm{~cm}$, size $>3.0 \mathrm{~cm}$ ), tumor extension (cerebellum, brainstem, ventricular and other). Histological characteristics (Pilocytic astrocytoma, anaplastic astrocytoma, ependymoma, glioblastoma, and other). WHO grade (WHO grade III and IV were assigned to high grade, WHO grade I and II were assigned to low grade). The extent of tumor resection (Local excision/biopsy, STR, GTR, and resection lobe of brain).

\section{Statistical Analyses}

The variables associated with OS in this study including age, race, sex, year at diagnosis, WHO grade, tumor size, histological type, and extent of surgery. We use the Kaplan-Meier method to assess overall survival rate, using the log-rank test to evaluate the differences between survival curves. Then we use the univariate and multivariate analyses to determine the effect prognostic factors on OS, both multivariate and univariate cox proportional hazard models were used to calculate HRs (hazard ratios) and the 95\% Cls (confidence intervals), If $P<0.05$ was considered statistically significant. Randomly dividing the population into validation group ( $n=152)$ and training group $(n=356)$. Then we use using the package of RMS in $\mathrm{R}$ version 4.1 .0 (http://www.r-project.org/) building a nomogram model to calculate 
risk score according to the formula. Calibration curve and Harrell's concordance index (C-index) were used to estimate the accuracy and consistency of nomogram between the predicted and actual OS rate.

\section{RESULTS}

\section{Patient Population and Baseline Characteristics}

A total of 508 patients were included in this study. Demographic information, surgery information and tumor characteristics were described in Table 1.

TABLE 1 | Patient demographics, tumor characteristics and treatment of 508 patients with histologically confirmed cerebellar gliomas

\section{Characteristics}

POPULATION SIZE

AGE

$$
<20
$$

20-60

$>60$

RACE

White

Black

Other

SEX

Female

Male

YEAR

2011-2015

2004-2010

HIST

Glioblastoma

Ependymoma

Anaplastic ast

Pilocytic ast

Other

WHO GRADE

Low

High

Unknow

EXTENSION

Cerebellum

Brainstem

Ventricular

Other

TUMOR SIZE

$<3 \mathrm{~cm}$

\section{ALL}

508

290(57.1\%)

151(29.7\%)

67(13.2\%)

396(78.0\%)

$58(11.4 \%)$

$54(10.6 \%)$

252(49.6\%)

256(50.4\%)

252(49.6\%)

256(50.4\%)

$67(13.2 \%)$

36(7.1\%)

29(5.7\%)

325(64.0\%)

51(10.0\%)

$52(10.2 \%)$

$67(13,2 \%)$

389(76.6\%)

$381(75.0 \%)$

59(11.6\%)

$31(6.1 \%)$

$37(7.3 \%)$

161(31.7\%)

\section{Local} excision/biopsy

24(4.7\%)

STR

GTR

152(29.9\%) 174(34.3\%)

$5(20.8 \%)$

$4(16.7 \%)$

$15(62.5 \%)$

$17(70.8 \%)$

$1(4.2 \%)$

$6(25.0 \%)$

$13(54.2 \%)$

$11(45.8 \%)$

$16(66.7 \%)$

$8(33.3 \%)$

$3(12.5 \%)$

$1(4.2 \%)$

$9(37.5 \%)$

$4(16.7 \%)$

$7(29.2 \%)$

$1(4.2 \%)$

$11(45.8 \%)$

$12(50.0 \%)$

$17(70.8 \%)$

$4(16.7 \%)$

$1(4.2 \%)$

$2(8.3 \%)$

$16(66.7 \%)$
$66(43.4 \%) \quad 121(69.5 \%)$

$62(40.8 \%) \quad 39(22.4 \%)$

$24(15.8 \%) \quad 14$ (8.0\%)

$122(80.3 \%) \quad 131(75.3 \%)$

$15(9.9 \%) \quad 25(14.4 \%)$

$15(9.9 \%) \quad 18(10.3 \%)$

$83(54.6 \%) \quad 78(44.8 \%)$

$69(45.4 \%) \quad 96(55.2 \%)$

$91(59.9 \%) \quad 142(81.6 \%)$

$61(40.1 \%) \quad 32(18.4 \%)$

$31(20.4 \%) \quad 17(9.8 \%)$

$9(5.9 \%) \quad 15(8.6 \%)$

$10(6.6 \%)$

$6(3.4 \%)$

$86(56.6 \%)$

$129(74.1 \%)$

$16(10.5 \%)$

$7(4.0 \%)$

$16(10.5 \%)$

$7(4.0 \%)$

$25(16.4 \%)$

$18(10.3 \%)$

$111(73.0 \%)$

149 (85.6\%)

$105(69.1 \%)$

$137(78.7 \%)$

$20(13.2 \%)$

$16(9.2 \%)$

$9(5.9 \%)$

$9(5.2 \%)$

$18(11.8 \%)$

$12(6.9 \%)$

$55(36.2 \%)$

$42(24.1 \%)$

\section{Resection lobe of brain $\mathrm{P}$-value}

158(31.1\%)

$<0.001$

$98(62.0 \%)$

$46(29.1 \%)$

$14(8.9 \%)$

0.221

$126(79.7 \%)$

$17(10.8 \%)$

$15(9.5 \%)$

0.346

$78(49.4 \%)$

$80(50.6 \%)$

$<0.001$

$3(1.9 \%)$

$155(98.1 \%)$

$<0.001$

$16(10.1 \%)$

$11(7.0 \%)$

$4(2.5 \%)$

$106(67.1 \%)$

$21(13.3 \%)$

$<0.001$

$28(17.7 \%)$

$13(8.2 \%)$

$117(74.1 \%)$

0.213

$122(77.2 \%)$

19 (12.0\%)

$12(7.6 \%)$

$5(3.2 \%)$

$<0.001$

$48(30.4 \%)$ 


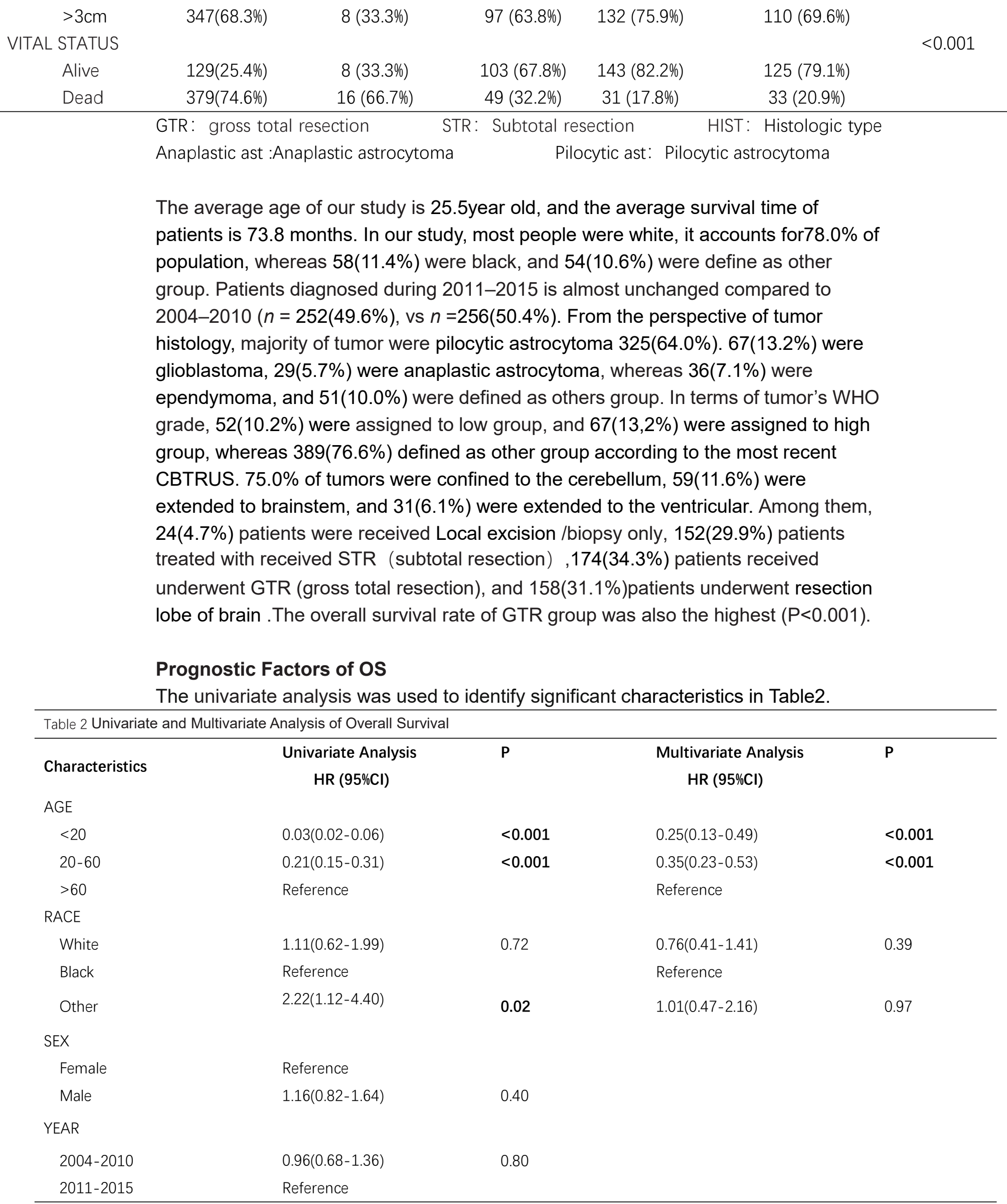




\begin{tabular}{|c|c|c|c|c|}
\hline \multicolumn{5}{|l|}{ HIST } \\
\hline Glioblastoma & 1.93(1.19-3.12) & 0.007 & $4.92(2.55-9.46)$ & $<0.001$ \\
\hline Ependymoma & $0.23(0.11-0.47)$ & $<0.001$ & $0.78(0.33-1.84)$ & 0.57 \\
\hline Anaplastic astrocytoma & Reference & & Reference & \\
\hline Pilocytic astrocytoma & $0.02(0.01-0.04)$ & $<0.001$ & $0.119(0.04-0.29)$ & $<0.001$ \\
\hline Other & $0.34(0.19-0.62)$ & $<0.001$ & $1.06(0.50-2.24)$ & 0.87 \\
\hline \multicolumn{5}{|l|}{ WHO GRADE } \\
\hline Low & $0.08(0.03-0.19)$ & $<0.001$ & $0.30(0.12-0.79)$ & 0.015 \\
\hline High & Reference & & Reference & \\
\hline Unknow & $0.14(0.09-0.20)$ & $<0.001$ & $0.43(0.27-0.68)$ & $<0.001$ \\
\hline \multicolumn{5}{|l|}{ EXTENSION } \\
\hline Cerebellum & $0.45(0.28-0.71)$ & $<0.001$ & $0.60(0.37-0.97)$ & 0.04 \\
\hline Brainstem & Reference & & Reference & \\
\hline Ventricular & $0.78(0.37-1.65)$ & 0.52 & $1.19(0.57-2.58)$ & 0.66 \\
\hline Other & $1.40(0.76-2.57)$ & 0.28 & $1.00(0.53-1.91)$ & 0.99 \\
\hline \multicolumn{5}{|l|}{ TUMOR SIZE } \\
\hline$<3 \mathrm{~cm}$ & Reference & & & \\
\hline$>3 \mathrm{~cm}$ & $0.78(0.55-1.12)$ & 0.18 & & \\
\hline \multicolumn{5}{|l|}{ SURGERY } \\
\hline Local excision/biopsy & $6.23(3.40-11.41)$ & $<0.001$ & $2.18(1.10-4.29)$ & 0.02 \\
\hline STR & $2.02(1.28-3.16)$ & 0.002 & $1.10(0.68-1.77)$ & 0.70 \\
\hline GTR & Reference & & Reference & \\
\hline Resection lobe of brain & $1.13(0.69-1.84)$ & 0.64 & $1.05(0.63-1.77)$ & 0.84 \\
\hline
\end{tabular}

including age, race, histologic type, tumor WHO grade, tumor extension, and surgery. Kaplan-Meier curves were painted to compare the overall survival cerebellar glioma by different variates as showed in Figure2. 
Figure2
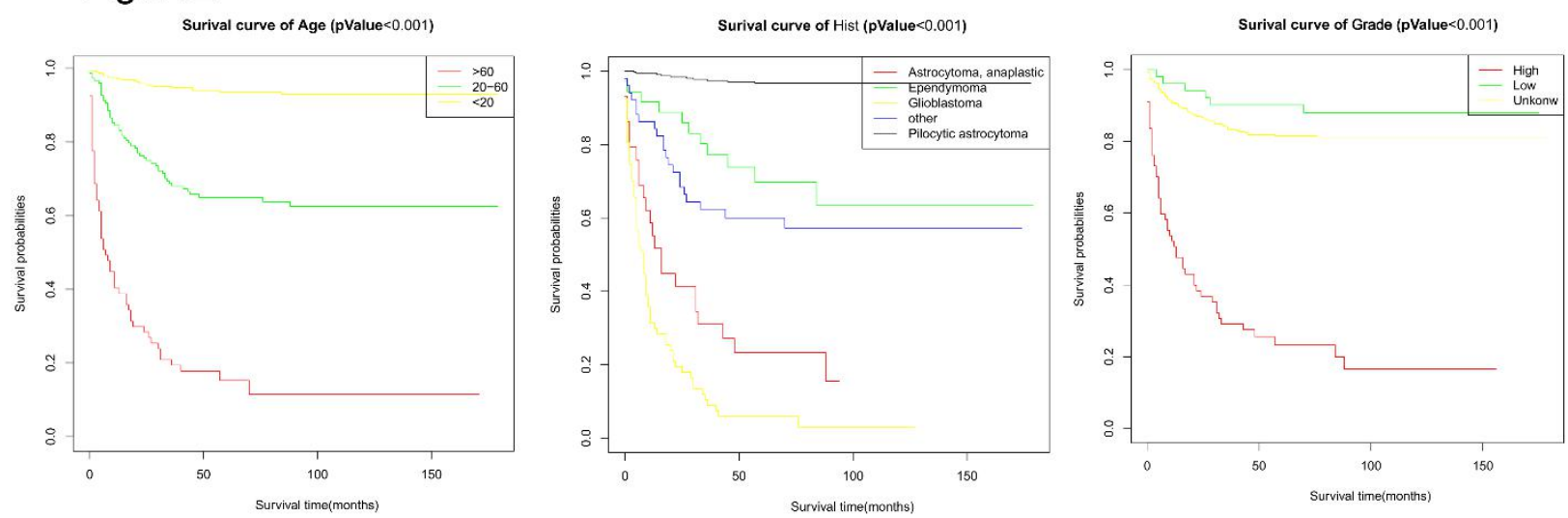

A

B
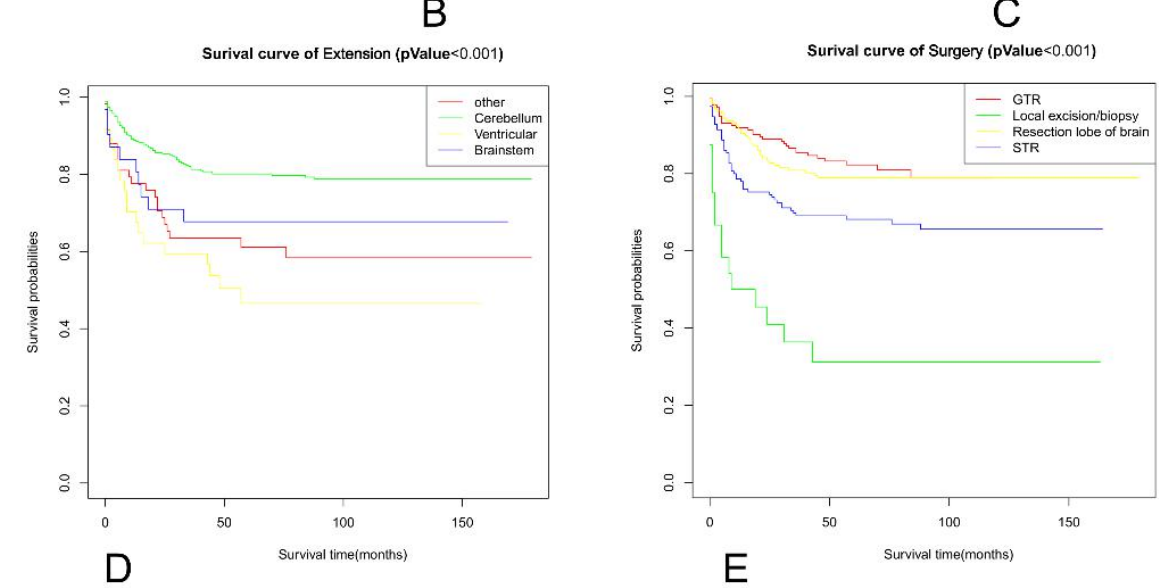

Figure 2. Kaplan-Meier curves for patients with cerebellum glioma by different variates. (A) Age, (B) Histology, (C) WHO Grade, (D)Extension, (E) Surgery.

The result showed that age $(P<0.001)$, histology type $(P<0.001)$, WHO grade $(P<$ $0.001)$, surgery $(P<0.001)$, these factors are related to the overall survival of the patients. Next, the nomograms model was build based on the five characteristics: age, race, extension, tumor grade, histological type, and surgery as showed in Figure 3. 


\section{Figure 3}

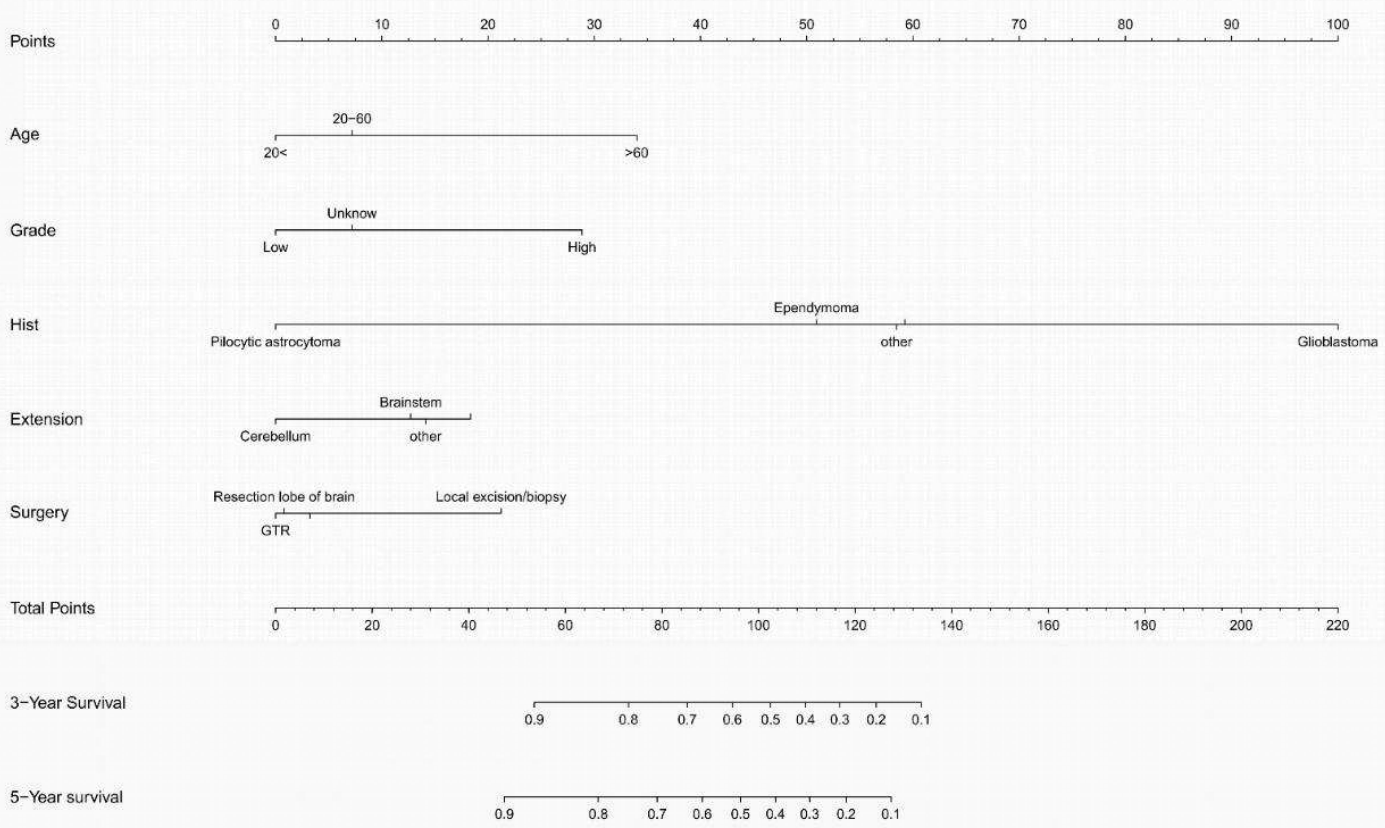

Figure 3. Nomogram for overall survival rate

The 3-year and 5-year overall survival rate were evaluated by nomogram to compute the corresponding score. Then, we randomly divide the data into Validation group and training group. Among them, there are 356 people in the training group and 152 people in the validation group. There has no significant statistical difference between the two groups (Table 3).

\begin{tabular}{|c|c|c|c|c|}
\hline Characteristics & All $n(\%)$ & Training $n(\%)$ & Validation $n(\%)$ & $p$ \\
\hline AGE & & & & 0.586 \\
\hline$<20$ & $290(57.1 \%)$ & 198 & 92 & \\
\hline $20-60$ & $151(29.7 \%)$ & 109 & 42 & \\
\hline$>60$ & $67(13.2 \%)$ & 49 & 18 & \\
\hline RACE & & & & 0.405 \\
\hline White & $396(78.0 \%)$ & 273 & 123 & \\
\hline Black & $58(11.4 \%)$ & 45 & 13 & \\
\hline Other & $54(10.6 \%)$ & 38 & 16 & \\
\hline SEX & & & & 0.373 \\
\hline Female & $252(49.6 \%)$ & 172 & 80 & \\
\hline Male & $256(50.4 \%)$ & 184 & 72 & \\
\hline YEAR & & & & 0.615 \\
\hline 2004-2010 & $252(49.6 \%)$ & 182 & 74 & \\
\hline
\end{tabular}


HIST

Glioblastoma

67(13.2\%)

50

Ependymoma

$36(7.1 \%)$

Anaplastic astrocytoma

29(5.7\%)

Pilocytic astrocytoma

$325(64.0 \%)$

51(10.0\%)

WHO GRADE

Low

$52(10.2 \%)$

High

$67(13,2 \%)$

46

Unknow

$389(76.6 \%)$

268

0.300

EXTENSION

Cerebellum

$381(75.0 \%)$

268

Brainstem

Ventricular

59(11.6\%)

41

$31(6.1 \%)$

Other

$37(7.3 \%)$

25

22

$<3 \mathrm{~cm}$

161(31.7\%)

115

$347(68.3 \%)$

241

SURGERY

Local excision/biopsy

STR

GTR

C-index (receiver operating curve) and internal calibration curve were plotted to verify the accuracy and discriminative of the model. The $\mathrm{C}$-index of overall survival rate prediction was0.909 (95\% Cl, (0.880-0.938)) and 0.932(95\% Cl, (0.889-0.975)), respectively. The ROC (receiver operating curve) and AUC (area under curve) were plotted. The area under curve values of the 3 year- and 5 -year overall survival rates in the training group were 0.935 and 0.941 , and in the validation group were 0.966 and 0.949 (Figure 4), which suggested that the predictive model was effective and accurate. The calibration curve of overall survival rate at 3 year- and 5-year shows that there was an excellent consistency between the predicted survival probability and the actual survival probability (Figure 5). 
Figure 4

3-year Survival AUC $=0.935$

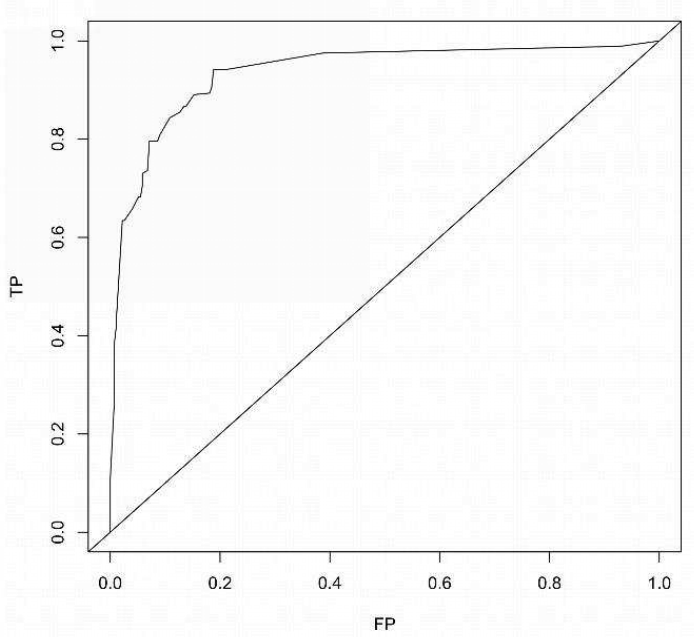

A $\quad$ 5-year Survival AUC $=0.941$

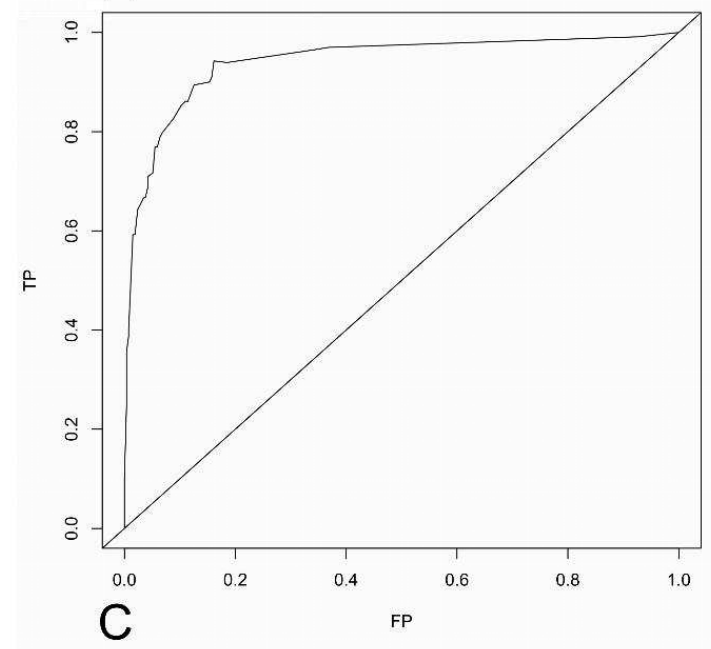

3-year Survival AUC $=0.966$

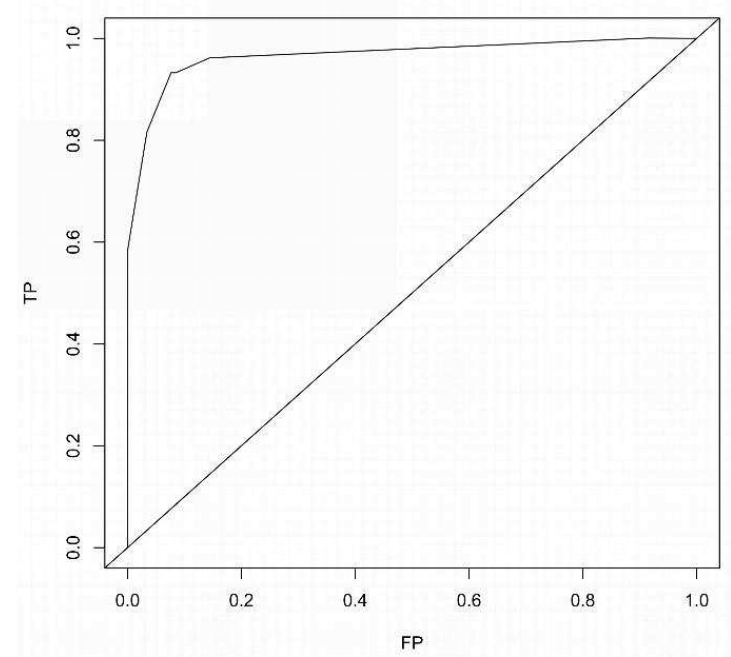

B 5-year Survival AUC $=0.949$

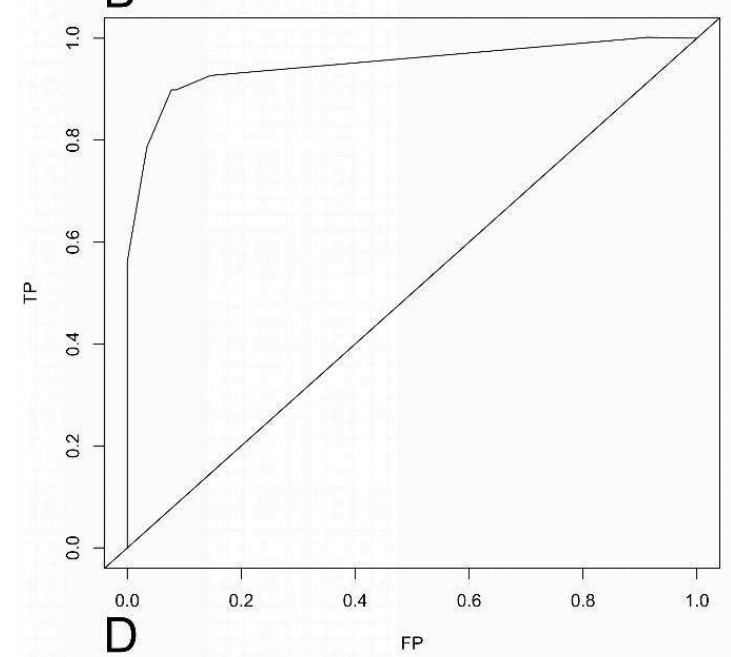

Figure 4. Time-dependent ROC curve and areas under ROC curve. Areas under ROC curve of 3-year overall survival rates in training group (A) and validation group (B). Areas under ROC curve of 5 -year overall survival rates in training group (C) and validation group (D). 


\section{Figure 5}

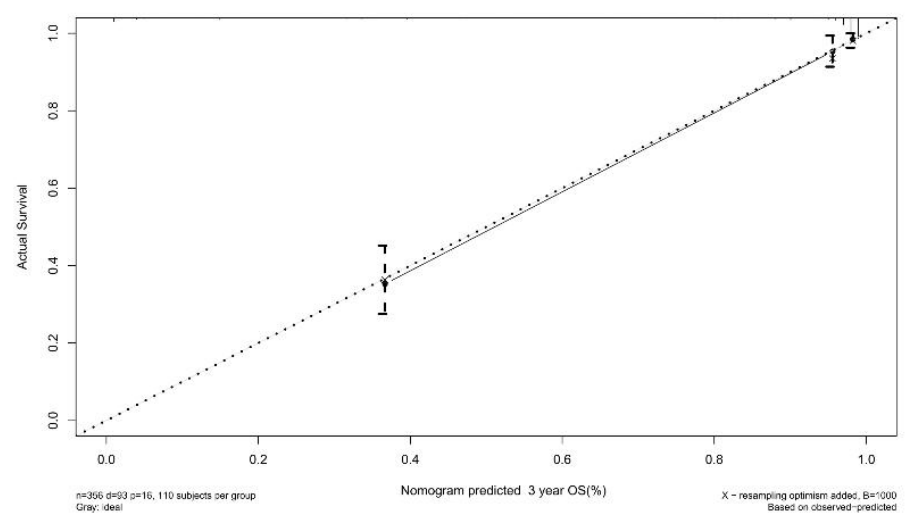

A

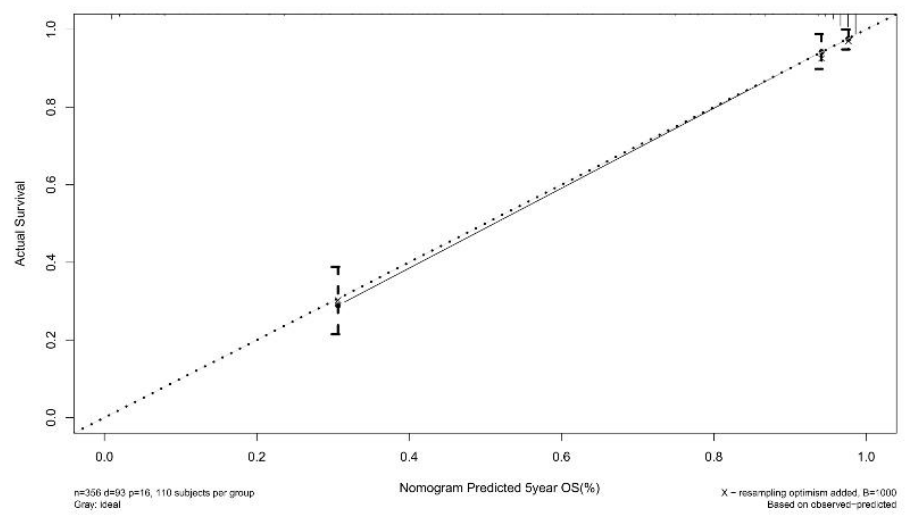

C

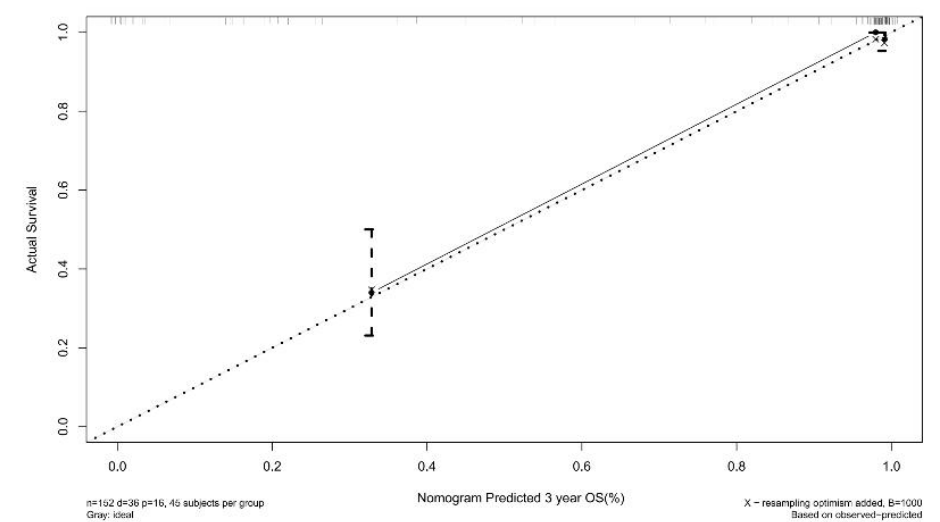

B

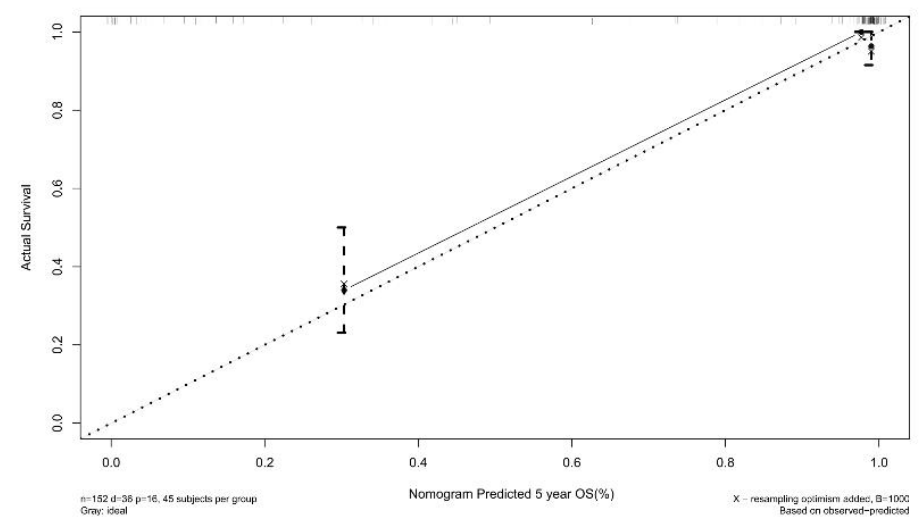

$\mathrm{D}$

Figure 5. Internal calibration plots of 3-year overall survival rates in training group (A) and validation group (B). Internal calibration plots of 5-year overall survival rates in training group (C) and validation group (D)

\section{Discussion}

CNS (Central nervous system) tumors comprise a majority of malignant and benign neoplasms prevalent across the entire age spectrum ${ }^{6}$. Although the incidence of central nervous system tumors is higher in adults, it is also the main cause of death in children ${ }^{7}$. The cerebellar glioma is a common type of central nervous system tumor in children but relatively rare in adults ${ }^{1}$. Since the incidence of cerebellar glioma is not high, there are not many studies focus on this area. Central nervous system tumors, especially tumors of the cerebellum and brainstem causing the greatest proportion of deaths $(37.9 \% \text { and } 16.6 \% \text {, respectively })^{8}$. Patients with cerebellar glioma often present with the symptom of intracranial hypertension, and some cerebellar symptoms for example ataxia in walking, unstable standing, dizziness, mental disorders and other symptoms that seriously affect the quality of daily life $e^{2,9-13}$. Our analysis of patients with cerebellar glioma demonstrated that the the younger the patient with cerebellar glioma, the better the prognosis, and age is an important factor affecting the prognosis. The difference in the prognosis of children and adults may be 
caused by differences in the molecular mechanism of tumors, but our study did not include the study of molecular mechanisms, because there is no relevant information about molecular mechanisms in the seed database. Ranjith Babu et al found that: Patients older than 40 years has poor outcomes, and age over 65 years had worst survival rate (4 versus 12 months, $p<0.0001$ ) compared to the younger patients ${ }^{14}$. Our study found that the prognosis of patients with cerebellar glioma is independent of gender. In terms of races, univariate analysis shows that there has no difference in the prognosis of whites and blacks, and the prognosis of the rest of the population is worse, but the difference was disappeared after multivariate analysis. Zhuoyi Liu reported that whether in univariate analysis or multivariate analysis: the prognosis of tumors has nothing to do with gender and race ${ }^{15}$. Our study found that cerebellar glioblastoma had the worst prognosis, followed by anaplastic astrocytoma, and pilocytic astrocytoma had the best prognosis. These differences were statistically significant regardless of univariate analysis or multivariate analysis. Glioblastoma has a high degree of malignancy, its growth rate is blocky, the boundary is not clear, and the operation is difficult to complete, which is the main reason for the poor prognosis. Xingwang Zhou et al demonstrates that oligodendroglioma, anaplastic glioma and glioblastoma were risk factors of early mortality for children, and pointed out that glioblastoma increases early childhood mortality ${ }^{16}$. Ankush Chandra et al found a very meaningful study: tumor $>40 \mathrm{~mm}$ were associated with decreased survival for patients with supratentorial glioblastoma ${ }^{17}$. But in our study, the prognosis of cerebellar glioma has little to do with the size of the tumor. This may be because there is no obvious correlation between the size and the degree of malignancy of the tumor. In this study, we found that the higher the WHO grade of the tumor, the worse the prognosis, the lower the patient's median survival time and overall survival rate. Adams et al found that the prognosis of high-grade gliomas was much worse than that of low-grade gliomas ${ }^{18}$. Some scholars found that the higher the grade of glioma, the higher the activity level and the higher the tumor angiogenesis ${ }^{19}$. Also, malignant high-grade gliomas are diffuse and infiltrating lesions that often infiltrate some important peripheral functional areas, which seriously affect the quality of life of patients ${ }^{20}$. In terms of the extension of the tumor, tumors confined to the cerebellum have the best prognosis, and the prognosis is poor after expansion to the brainstem. It may be that after the tumor has expanded to the brainstem, it is difficult to completely removed, because the brainstem is the core part of the brain. Weber et al found that extent of surgery and brainstem involvement were associated with poorer survival ${ }^{14}$. The conclusion is roughly the same as ours. As for extent of tumor resection, univariate analysis showed that patients with total tumor resection had the best prognosis and the highest overall survival rate. Univariate analysis showed that patients with total tumor resection had the best prognosis and the highest overall survival rate. However, in a multivariate analysis, the overall survival rate of the patient has little to do with the degree of tumor resection. Our research also has many limitations. First of all, the data available in SEER is observational. The allocation of subjects is arbitrary and lacks randomization. Secondly, we do not have the authority to obtain information about radiotherapy and chemotherapy. Currently, chemotherapy is considered to be 
the standard treatment for patients with the following diseases: high-grade glioma in the United States. In addition, other important factors are not easy available in the SEER database, which includes the patient's preoperative symptoms, imaging data, family financial status, postoperative neurological function, and the patient's postoperative quality of life. Our research is a retrospective clinical study, with the general limitations of retrospective studies. Therefore, it is necessary to conduct high-quality prospective studies to verify our conclusions.

\section{Conclusion}

Our study is a large-scale population-based study of cerebellar glioma. It directly compares factors such as age, gender, tumor size, histological type, grade, and resection, founding that young age, pilocytic astrocytoma, low-grade, and confined tumor, can significantly prolong the survival time of patients with cerebellar glioma while cerebellar glioblastoma can significantly reduce the survival time of the patients. Our study provides very useful information for further research on cerebellar glioma.

\section{References}

1 Guerreiro Stucklin, A. S. \& Grotzer, M. A. Cerebellar tumors. Handbook of clinical neurology 155, 289-299, doi:10.1016/b978-0-444-64189-2.00019-6 (2018).

2 Hegedüs, K. \& Molnár, P. Primary cerebellar glioblastoma multiforme with an unusually long survival. Case report. Journal of neurosurgery 58, 589-592, doi:10.3171/jns.1983.58.4.0589 (1983).

3 Nishioka, H., Saito, F., Haraoka, J. \& Miwa, T. [Glioblastoma of the cerebellum: report of an autopsy case associated with intratumoral hemorrhage and CSF seedings]. No shinkei geka. Neurological surgery 19, 547-552 (1991).

4 Kopelson, G. \& Linggood, R. Infratentorial glioblastoma: the role of neuraxis irradiation. International journal of radiation oncology, biology, physics 8, 999-1003, doi:10.1016/0360-3016(82)90167-5 (1982).

5 Salazar, O. M. Primary malignant cerebellar astrocytomas in children: a signal for postoperative craniospinal irradiation. International journal of radiation oncology, biology, physics 7, 1661-1665, doi:10.1016/0360-3016(81)90189-9 (1981).

6 Louis, D. N. et al. The 2016 World Health Organization Classification of Tumors of the Central Nervous System: a summary. Acta neuropathologica 131, 803-820, doi:10.1007/s00401-016-1545-1 (2016).

7 Ostrom, Q. T. et al. CBTRUS Statistical Report: Primary Brain and Other Central Nervous System Tumors Diagnosed in the United States in 2012-2016. Neuro-oncology 21, v1-v100, doi:10.1093/neuonc/noz150 (2019).

8 Ostrom, Q. T. et al. CBTRUS Statistical Report: Primary Brain and Other Central Nervous System Tumors Diagnosed in the United States in 2009-2013. Neuro-oncology 18, v1-v75, doi:10.1093/neuonc/now207 (2016).

9 Aun, R. A., Stavale, J. N. \& Silva Junior, D. [Glioblastoma multiforme of the cerebellum. Report of a case]. Arquivos de neuro-psiquiatria 39, 350-354, doi:10.1590/s0004-282×1981000300013 (1981).

10 Kulkarni, A. V., Becker, L. E., Jay, V., Armstrong, D. C. \& Drake, J. M. Primary cerebellar 
glioblastomas multiforme in children. Report of four cases. Journal of neurosurgery 90 , 546-550, doi:10.3171/jns.1999.90.3.0546 (1999).

11 Mattos, J. P. et al. Cerebellar glioblastoma multiforme in an adult. Arquivos de neuro-psiquiatria 64, 132-135, doi:10.1590/s0004-282×2006000100028 (2006).

12 Rosenfeld, J., Rossi, M. L. \& Briggs, M. Glioblastoma multiforme of the cerebellum in an elderly man. A case report. Tumori 75, 626-629 (1989).

13 Occhiogrosso, M., Spada, A., Merlicco, G., Vailati, G. \& De Benedictis, G. Malignant cerebellar astrocytoma. Report of five cases. Journal of neurosurgical sciences $\mathbf{2 9}, \mathbf{4 3 - 5 0}$ (1985).

14 Weber, D. C. et al. Outcome and prognostic factors in cerebellar glioblastoma multiforme in adults: a retrospective study from the Rare Cancer Network. International journal of radiation oncology, biology, physics 66, 179-186, doi:10.1016/j.ijrobp.2006.04.035 (2006).

15 Liu, Z. et al. The Epidemiological Characteristics and Prognostic Factors of Low-Grade Brainstem Glioma: A Real-World Study of Pediatric and Adult Patients. Frontiers in oncology 10, 391, doi:10.3389/fonc.2020.00391 (2020).

16 Zhou, X. et al. Pediatric Glioma Outcomes: Predictors of Early Mortality. World neurosurgery 139, e700-e707, doi:10.1016/j.wneu.2020.04.107 (2020).

17 Chandra, A. et al. Comparative Analysis of Survival Outcomes and Prognostic Factors of Supratentorial versus Cerebellar Glioblastoma in the Elderly: Does Location Really Matter? World neurosurgery 146, e755-e767, doi:10.1016/j.wneu.2020.11.003 (2021).

18 Adams, H. et al. Adult cerebellar glioblastoma: understanding survival and prognostic factors using a population-based database from 1973 to 2009. World neurosurgery 80 , e237-243, doi:10.1016/j.wneu.2013.02.010 (2013).

19 Zhao, M. et al. Quantitative analysis of permeability for glioma grading using dynamic contrast-enhanced magnetic resonance imaging. Oncology letters 14, 5418-5426, doi:10.3892/ol.2017.6895 (2017)

20 Simon, J. E. et al. Synergistic Therapies for Recurrent Malignant Gliomas. World neurosurgery 133, 237-239, doi:10.1016/j.wneu.2019.10.033 (2020). 